\title{
Almost periodic solutions of differential equations with piecewise constant argument of generalized type
}

\author{
M. U. Akhmet* \\ Department of Mathematics and Institute of Applied Mathematics, Middle East Technical \\ University, 06531 Ankara, Turkey \\ marat@metu.edu.tr
}

\begin{abstract}
We consider existence and stability of an almost periodic solution of the following hybrid system

$$
\frac{d x(t)}{d t}=A(t) x(t)+f\left(t, x\left(\theta_{\beta(t)-p_{1}}\right), x\left(\theta_{\beta(t)-p_{2}}\right), \ldots, x\left(\theta_{\beta(t)-p_{m}}\right)\right),
$$

where $x \in \mathbb{R}^{n}, t \in \mathbb{R}, \beta(t)=i$ if $\theta_{i} \leq t<\theta_{i+1}, i=\ldots-2,-1,0,1,2, \ldots$, is an identification function, $\theta_{i}$ is a strictly ordered sequence of real numbers, unbounded on the left and on the right, $p_{j}, j=1,2, \ldots, m$, are fixed integers, and the linear homogeneous system associated with (11) satisfies exponential dichotomy. The deviations of the argument are not restricted by any sign assumption when existence is considered. The problem of positive (almost periodic) solutions of the logistic equation is discussed as an example. A new technique of investigation of equations with piecewise argument, based on integral representation, is developed.
\end{abstract}

Key words and phrases: Quasilinear system; Almost periodic solutions; Piecewise constant argument of general type; Advanced-delayed argument; Differential logistic equations; Positive solutions.

1991 Mathematics Subject Classification: 34K14; 34K20.

${ }^{*}$ M.U. Akhmet is previously known as M. U. Akhmetov. 


\section{Introduction and Preliminaries}

The theory of differential equations with piecewise constant argument (EPCA) of the type

$$
\frac{d x(t)}{d t}=f\left(t, x\left(\left[t-p_{1}\right]\right), x\left(\left[t-p_{2}\right]\right), \ldots, x\left(\left[t-p_{m}\right]\right)\right)
$$

where [.] signifies the greatest integer function, was initiated in [13, 30] and developed by many authors [1, 8, 9, 25, 28, 29], 32, 36]-39]. These systems have been under intensive investigation for the last twenty years. They describe hybrid dynamical systems and combine properties of both differential and difference equations. An example of the application of these equations to the problems of biology can be found in [11]. One of the novel ideas in our paper is that system (II) is of general type (EPCAG) for equation (2). Indeed if we take $\theta_{i}=i, i=\ldots,-2,-1,0,1,2, \ldots$ then (11) takes the form of (2).

The existing method of investigation of EPCA, as it was proposed by its founders [13, 30], is based on the reduction of EPCA to discrete equations, and it has been the only method to prove assertions about EPCA until now. In our paper, we (apparently for the first time) propose another approach to the problem. In fact, we are dealing with the construction of an equivalent integral equation. Since we do not need additional assumptions on the reduced discrete equations for investigating EPCAG, the new method requires more easily verifiable conditions, similar to those for ordinary differential equations. So, solving the problems of EPCAG (as well as of EPCA) may become less cumbersome if the approach proposed in our paper is applied.

Another novelty in our investigation is that we consider equations with deviated argument of mixed (advanced-delayed) type. Even in the case of advanced argument, there are certain difficulties if we try to define a solution for increasing $t$ [22]. J. Hale remarked in [18] that "these equations (of mixed type) seem to dictate that boundary conditions should be specified in order to obtain a solution in the way as one does for elliptic partial differential equations. "We regard the boundedness of the solution on $R$ as a boundary condition in our investigation. Similar arguments were used in 6, 12, 16, to investigate various problems for ordinary and functional differential equations.

The existence of almost periodic solutions is one of the most interesting subjects of 
the theory of differential equations (see, for example, [2, 14, 21] and the references cited there). This problem has been considered in the context of EPCA in many papers, such as [32, 38, 40].

To solve the problems of the present paper, we intend to apply our knowledge about the almost periodicity of discontinuous solutions of impulsive systems [3]-[5], [17, 31, 34]. One should not be surprised with the relation between EPCAG and impulsive differential equations. This possibility was mentioned in [13] for EPCA, and in [20] for differential equations with discontinuous right hand side.

Original ideas on the spaces of discontinuous functions are to be found in [17, 23. 33, 35]. Following these results, in [4] we introduced Bohner type discontinuous almost periodic functions using topology as well as metric, in the spaces of discontinuous functions and in the discrete spaces of sets of points on the real axes, unbounded on the left and on the right. The multiplicity of one for the elements of the sets was mentioned there, since we supposed that the distances between neighbors are uniformly separated from zero. Further our proposals based on the metric were developed in Supplement $A$ of [31. In the present paper we again consider the spaces from the topological point of view, assuming that the multiplicity more or equal to one.

One can be confident that the reduction to integral equations, as well as the awareness about the theory of discontinuous functions, can diminish the number of "strange "properties of EPCA, which are usually generated by the reduction to difference equations, and can give explanations of certain phenomena. For example, the result on the module containment considered in 38] becomes less specific if one compares it with our Theorem [6 (see also Example 1). But we should note that the reduction to discrete equations is preferable in some cases, as in [19, 26, 27], where the period-doubling bifurcation and the generation of chaos by a logistic EPCA are considered.

Let $\mathbb{Z}, \mathbb{N}$, and $\mathbb{R}$ be the sets of all integers, natural and real numbers, respectively, and $\|\cdot\|$ be the euclidean norm in $\mathbb{R}^{n}, n \in \mathbb{N}$. Let $s \in R$ be a positive number. We denote $G_{s}=\left\{x \in \mathbb{R}^{n} \mid\|x\| \leq s\right\}$ and $G_{s}^{m}=G_{s} \times G_{s} \ldots \times G_{s}$ (that is, $G_{s}^{m}$ is an $m-$ times Cartesian product of $\left.G_{s}\right)$. Let a $C_{0}(R)$ (respectively $C_{0}\left(R \times G_{H}^{m}\right)$ for a given $H \in R, H>0$ ) be the set of all bounded and uniformly continuous functions on $R$ 
(respectively on $R \times G_{H}^{m}$ ). For $f \in C_{0}(R)$ (respectively $C_{0}\left(R \times G_{H}^{m}\right)$ ) and $\tau \in R$, the translate of $f$ by $\tau$ is the function $Q_{\tau} f=f(t+\tau), t \in R$ ( respectively $Q_{\tau} f(t, z)=$ $\left.f(t+\tau, z),(t, z) \in R \times G_{H}^{m}\right)$. A number $\tau \in R$ is called an $\epsilon-$ translation number of a function $f \in C_{0}(R)\left(C_{0}\left(R \times G_{H}^{m}\right)\right)$ if $\left\|Q_{\tau} f-f\right\|<\epsilon$ for every $t \in R\left((t, z) \in R \times G_{H}^{m}\right)$. A set $S \subset R$ is said to be relatively dense if there exists a number $l>0$, such that $[a, a+l] \cap S \neq \emptyset$ for all $a \in R$.

Definition 1 A function $f \in C_{0}(R)\left(C_{0}\left(R \times G_{H}^{m}\right)\right)$ is said to be almost periodic (almost periodic in $t$ uniformly with respect to $z \in G_{H}^{m}$ ) if for every $\epsilon \in R, \epsilon>0$, there exists a relatively dense set of $\epsilon$ - translation numbers of $\mathrm{f}$.

Denote by $\mathcal{A P}(R)\left(\mathcal{A P}\left(R \times G_{H}^{m}\right)\right)$ the set of all such functions .

The following assumptions will be needed throughout the paper.

$\left(C_{1}\right) A(t) \in \mathcal{A P}(R)$ is an $n \times n$ matrix;

$\left(C_{2}\right) f \in \mathcal{A P}\left(R \times G_{s}^{m}\right)$, for every $s \in R, s \geq 0$

$\left(C_{3}\right) \exists l \in R, l>0$, such that

$$
\left\|f\left(t, z_{1}\right)-f\left(t, z_{2}\right)\right\| \leq l \sum_{j=1}^{m}\left\|z_{1}^{j}-z_{2}^{j}\right\|,
$$

where $z_{i}=\left(z_{i}^{1}, \ldots, z_{i}^{m}\right) \in R^{n m}, i=1,2$.

Let

$$
\frac{d x}{d t}=A(t) x
$$

be the homogeneous linear system associated with (11), and $X(t)$ be a fundamental matrix of (3).

$\left(C_{4}\right)$ system (3) satisfies exponential dichotomy, that is, there exist a projection $P$ and positive constants $\sigma_{1}, \sigma_{2}, K_{1}, K_{2}$, such that

$$
\begin{aligned}
& \left\|X(t) P X^{-1}(s)\right\| \leq K_{1} \exp \left(-\sigma_{1}(t-s)\right), t \geq s \\
& \left\|X(t)(I-P) X^{-1}(s)\right\| \leq K_{2} \exp \left(\sigma_{2}(s-t)\right), t \leq s .
\end{aligned}
$$


Let $\Theta$ be a space of strictly ordered sequences $\left\{\theta_{i}\right\} \subset R, i \in \mathbb{Z}$, such that $\left|\theta_{i}\right| \rightarrow \infty$, if $|i| \rightarrow \infty$. Denote by $\mathcal{P C}$ the set of all functions from $\mathbb{R}$ to $\mathbb{R}^{n}$ that are piecewise continuous with discontinuities of the first type. Assume that the set of discontinuities of every function from $\mathcal{P C}$, numerated in a strict order, is an element of $\Theta$. Moreover, these functions are uniformly continuous on the set $\cup_{i \in \mathbb{Z}}\left(\theta_{i}, \theta_{i+1}\right)$, and they are left or right continuous at every point of discontinuity.

Denote by $\mathcal{P} \mathcal{C}_{r} \subset \mathcal{P C}$ the set of all continuous from the right functions. Similarly, one can define a set $\mathcal{P} \mathcal{C}_{l}$. If $\phi \in \mathcal{P C}$, then one can define a function $\phi_{r} \in \mathcal{P} \mathcal{C}_{r}$, such that $\phi_{r}(t)=\phi(t)$ everywhere, except possibly at points $t=\theta_{i}$, that is,

$$
\phi_{r}(t)= \begin{cases}\phi(t), & \text { if } t \neq \theta_{i} \\ \phi\left(\theta_{i}+\right), i \in Z, & \text { otherwise }\end{cases}
$$

We shall call the function $\phi_{r}(t)$ a right extension of the function $\phi(t) \in \mathcal{P C}$. Since the function $\beta(t)$ is right continuous, it is reasonable to consider only the space $\mathcal{P} \mathcal{C}_{r}$ in our paper, extending, to the right, if necessary, every function from $\mathcal{P C}$ that we obtain in our discussion. Since functions from $\mathcal{P C}$ are assumed to be the derivatives or limits of the solutions of EPCAG, no difficulty arises from this agreement. In what follows we assume that $\beta(t) \in \mathcal{P C}_{r}$. The following definition of a solution of EPCAG which is a slightly changed form of the corresponding definition for EPCA [28, 29] can be given.

Definition 2 A function $x(t)$ is a solution of (1) on $\mathbb{R}$ if:

(i) $x(t)$ is continuous on $\mathbb{R}$;

(ii) the derivative $x^{\prime}(t)$ exists at each point $t \in \mathbb{R}$, with the possible exception of the points $\theta_{i}, i \in \mathbb{Z}$, where one-sided derivatives exist;

(iii) equation (1) is satisfied on each interval $\left[\theta_{i}, \theta_{i+1}\right), i \in \mathbb{Z}$.

It is obvious that the derivative of a solution $x(t)$ is a function from $\mathcal{P} \mathcal{C}_{r}$, if we assume it to be the right derivative at $t=\theta_{i}, i \in \mathbb{Z}$.

Let

$$
G(t, s)=\left\{\begin{aligned}
X(t) P X^{-1}(s), & \text { if } t \geq s \\
X(t)(P-I) X^{-1}(s), & \text { if } t<s
\end{aligned}\right.
$$


be the Green's function of (3) . Denote

$$
F_{\theta}(\psi(t))=f\left(t, \psi\left(\theta_{\beta(t)-p_{1}}\right), \psi\left(\theta_{\beta(t)-p_{2}}\right), \ldots, \psi\left(\theta_{\beta(t)-p_{m}}\right)\right)
$$

where $\psi(t) \in C_{0}(R)$.

The following is one of the most important assertion for our method of investigation of EPCAG.

Lemma 1 A function $x(t) \in C_{0}(R)$ is a solution of (1) if and only if

$$
x(t)=\int_{-\infty}^{\infty} G(t, s) F_{\theta}(x(s)) d s .
$$

Proof.

Necessity. Assume that $x(t) \in C_{0}(R)$ is a solution of (11). Denote

$$
\phi(t)=\int_{-\infty}^{\infty} G(t, s) F_{\theta}(x(s)) d s
$$

By straightforward calculation we can see that the function $\phi(t)$ is bounded and continuous on $R$.

Assume that $t \neq \theta_{i}, i \in Z$. Then

$$
\phi^{\prime}(t)=A(t) \phi(t)+F_{\theta}(x(t))
$$

and

$$
x^{\prime}(t)=A(t) x(t)+F_{\theta}(x(t)) .
$$

Hence,

$$
[\phi(t)-x(t)]^{\prime}=A(t)[\phi(t)-x(t)]
$$

Calculating the limit values at $t=\theta_{j}, j \in \mathbb{Z}$, we find that

$$
\begin{aligned}
& \phi^{\prime}\left(\theta_{j} \pm 0\right)=A\left(\theta_{j} \pm 0\right) \phi\left(\theta_{j} \pm 0\right)+F_{\theta}\left(x\left(\theta_{j} \pm 0\right)\right) \\
& x^{\prime}\left(\theta_{j} \pm 0\right)=A\left(\theta_{j} \pm 0\right) x\left(\theta_{j} \pm 0\right)+F_{\theta}\left(x\left(\theta_{j} \pm 0\right)\right) .
\end{aligned}
$$


Consequently,

$$
\left.[\phi(t)-x(t)]^{\prime}\right|_{t=\theta_{j}+0}=\left.[\phi(t)-x(t)]^{\prime}\right|_{t=\theta_{j}-0} .
$$

Thus, $[\phi(t)-x(t)]$ is a continuously differentiable function on $R$, satisfying (3). That is, $[\phi(t)-x(t)]=0$ on $R$.

Sufficiency. Suppose that (4) is valid and $x(t) \in C_{0}(R)$. Fix $i \in \mathbb{Z}$ and consider the interval $\left[\theta_{i}, \theta_{i+1}\right)$. If $t \in\left(\theta_{i}, \theta_{i+1}\right)$, then by differentiating one can see that $x(t)$ satisfies (11). Moreover, considering $t \rightarrow \theta_{i}+$, and taking into account that $\beta(t)$ is a right-continuous function, we obtain that $x(t)$ satisfies (11) on $\left[\theta_{i}, \theta_{i+1}\right)$. The lemma is proved.

\section{Wexler sequences}

Fix $\theta \in \Theta$, and consider a sequence $\gamma_{i}, i \in \mathbb{Z}, \gamma_{i+1} \geq \gamma_{i}$, such that for every $\gamma_{i} \in \gamma$ there exists an element $\theta_{j} \in \theta$ such that $\gamma_{i}=\theta_{j}$. Let $m(i), i \in \mathbb{Z}$, be the number of elements of $\gamma$ which are equal to $\theta_{i}$. We shall call this number the multiplicity of $\theta_{i}$ with respect to $\gamma$. Denote $m(\gamma)=\sup _{i} m(i)$. Denote by $\Gamma$ the set of all sequences $\gamma$ such that $m(\gamma)<\infty$. If $\gamma \in \Gamma$, then we shall say that $m(\gamma)$ is the maximal multiplicity of $\gamma$. It is obvious that $\left|\gamma_{i}\right| \rightarrow \infty$ if $|i| \rightarrow \infty$, for every $\gamma \in \Gamma$, and that $\Theta \subset \Gamma$. We shall call $\theta$ a support of $\gamma$, and $\gamma$ a representative of $\theta$. Introduce the following distance $\left\|\gamma^{(1)}-\gamma^{(2)}\right\|=\sup _{i}\left\|\gamma_{i}^{(1)}-\gamma_{i}^{(2)}\right\|$ if $\gamma^{(1)}, \gamma^{(2)} \in \Gamma$. We shall say that elements $\theta^{(1)}, \theta^{(2)} \in \Theta$ are $\epsilon-$ equivalent and write $\theta^{(1)} \epsilon \theta^{(2)}$, if there exist the representatives $\gamma^{(1)}$ and $\gamma^{(2)}$ in $\Gamma$ of $\theta^{(1)}$ and $\theta^{(2)}$, respectively, such that $\left\|\gamma^{(1)}-\gamma^{(2)}\right\|<\epsilon$. Moreover, we shall say that these sequences are in the $\epsilon-$ neighborhoods of each other.

The topology defined on the basis of all $\epsilon-$ neighborhoods, $0<\epsilon<\infty$, of all elements of $\Theta$ is named as $B^{s}$ - topology. Obviously, it is a Hausdorff topology.

Lemma 2 If $\theta^{(1)} \epsilon_{1} \theta^{(2)}, \theta^{(2)} \epsilon_{2} \theta^{(3)}$, then $\theta^{(1)}\left(\epsilon_{1}+\epsilon_{2}\right) \theta^{(3)}$.

Proof. Let $\gamma^{(1)}, \gamma^{(3)}$ be the representatives of $\theta^{(1)}, \theta^{(3)}$, respectively, and $\gamma^{(2)}, \gamma^{(4)}$ be the representatives of $\theta^{(2)}$ such that $\left\|\gamma^{(1)}-\gamma^{(2)}\right\|<\epsilon_{1}$ and $\left\|\gamma^{(4)}-\gamma^{(3)}\right\|<\epsilon_{1}$. Let $m^{(2)}, m^{(4)}$ be the maximal multiplicities of $\gamma^{(2)}, \gamma^{(4)}$, respectively. Denote $m_{0}=\max \left(m^{(2)}, m^{(4)}\right)$ and define a representative $\bar{\gamma}^{(2)}$ of $\theta^{(2)}$ with multiplicity $m(i)=m_{0}, i \in \mathbb{Z}$. Enlarging, if necessary, the multiplicity of elements and shifting the indeces of elements by the same 
number, in accordance with the change from $\gamma^{(2)}$ to $\gamma^{(4)}$, we can construct representatives $\bar{\gamma}^{(1)}, \bar{\gamma}^{(3)}$ of $\theta^{(1)}, \theta^{(3)}$, respectively, such that $\left|\bar{\gamma}_{i}^{(2)}-\bar{\gamma}_{i}^{(1)}\right|<\epsilon_{1},\left|\bar{\gamma}_{i}^{(2)}-\bar{\gamma}_{i}^{(3)}\right|<\epsilon_{2}, i \in \mathbb{Z}$. Consequently, $\left\|\bar{\gamma}^{(3)}-\bar{\gamma}^{(1)}\right\|<\epsilon_{1}+\epsilon_{2}$. The lemma is proved.

Let $a_{i}, i \in \mathbb{Z}$, be a sequence in $\mathbb{R}^{n}$. An integer $p$ is called an $\epsilon$-almost period of the sequence, if $\left\|a_{i+p}-a_{i}\right\|<\epsilon$ for any $i \in \mathbb{Z}$.

Definition $3 A$ sequence $a_{i}, i \in \mathbb{Z}$, is almost periodic, if for any $\epsilon>0$ there exists a relatively dense set of its $\epsilon$-almost periods.

Let $\gamma \in \Gamma, i, j \in \mathbb{Z}$. Denote $\gamma_{i}^{j}=\gamma_{i+j}-\gamma_{i}$ and define sequences $\gamma^{j}=\left\{\gamma_{i}^{j}\right\} \in \Gamma, j \in \mathbb{Z}$.

Definition 4 [17, 31] Sequences $\gamma^{j}, j \in \mathbb{Z}$, are equipotentially almost periodic if for an arbitrary $\epsilon>0$ there exists a relatively dense set of $\epsilon-$ almost periods that are common for all $\gamma^{j}, j \in \mathbb{Z}$.

Definition 5 We shall say that $\theta \in \Theta$ is a Wexler sequence, if there exists a representative $\gamma$ of $\theta$ with equipotentially almost periodic $\gamma^{j}, j \in \mathbb{Z}$.

Let $\gamma \in \Gamma, \epsilon>0$, be given. Denote by $T_{\epsilon} \subset R$ the set of numbers $\tau$, for which there exists at least one number $q_{\tau} \in \mathbb{Z}$, such that

$$
\left|\gamma_{i}^{q_{\tau}}-\tau\right|<\epsilon, i \in \mathbb{Z}
$$

Denote by $Q_{\tau}$ the set of all numbers $q_{\tau}$ satisfying (6) for fixed $\epsilon$ and $\tau$, and $Q_{\epsilon}=$ $\bigcup_{\tau \in T_{\epsilon}} Q_{\tau}$. The following lemmas were proved in [17, 35] for $m(\gamma)=1$. But one can easily, repeating the proof in [17, to verify that they valid if $1<m(\gamma)<\infty$. For example, equivalence of conditions $(a)$ and $(b)$ of Lemma 3 is considered in 31.

Lemma 3 The following statements are equivalent

(a) the sequences $\gamma^{j}, j \in \mathbb{Z}$, are equipotentially almost periodic;

(b) the set $T_{\epsilon}$ is relatively dense for any $\epsilon>0$;

(c) the set $Q_{\epsilon}$ is relatively dense for any $\epsilon>0$. 
Lemma 4 Assume that sequences $\gamma^{j}, j \in \mathbb{Z}$, are equipotentially almost periodic. Then for arbitrary $l>0$ there exists $n_{0} \in N$, such that any interval of length $l$ contains at most $n_{0}$ elements of $\gamma$.

Fix $\theta \in \Theta$ and let $h=\left\{h_{n}\right\}, n \in \mathbb{N}$, be a sequence of real numbers. Assume that the sequence of shifts $\left\{\theta+h_{n}\right\}_{n}$ is convergent in $B^{s}-$ topology. We shall denote the limit element as $Q_{h} \theta$.

Definition 6 An element $\theta \in \Theta$ has the Bohner property, if every sequence $\left\{h_{n}^{\prime}\right\}$ contains a subsequence $h \subset h^{\prime}$, such that $Q_{h} \theta$ exists.

Theorem 1 If $\theta \in \Theta$ is a Wexler sequence, then it satisfies the Bohner property and $Q_{h} \theta$ is a Wexler sequence for arbitrary $h$.

Proof. Let $\theta$ be a Wexler sequence and $\gamma^{j}, j \in \mathbb{Z}$, be equipotentially almost periodic, where $\gamma$ is the representative of $\theta$. The almost periodicity of $\gamma^{1}$ implies that there exists $\kappa>0$, such that $0 \leq \theta_{i}^{1}<\kappa, i \in \mathbb{Z}$. Hence, for arbitrary $n \in N$ there exists $i_{n}$ such that $\gamma_{i_{n}}+h_{n} \in[0, \kappa]$. Denote $\gamma^{(n)}=\left\{\gamma_{i+i_{n}}\right\}_{i}$. Clearly, $\gamma^{(n)} \in \Gamma, m\left(\gamma^{(n)}\right)=m(\gamma)$, and $\gamma^{(n) j}, j \in \mathbb{Z}$, are equipotentially almost periodic.

Using Theorem 1, p. 129 [17] and the equipotentially almost periodicity of $\gamma^{(n) j}$, one can show that there exists a subsequence $n_{k}$, let us say it is $n$ itself, such that for arbitrary $\epsilon>0$ there exists $n(\epsilon) \in N$, such that

$$
\left\|\gamma^{(m) j}-\gamma^{(p) j}\right\|<\frac{\epsilon}{2}, j \in \mathbb{Z}, \text { if } m, p>n(\epsilon) .
$$

Moreover, without loss of generality, we assume that $\gamma_{0}^{(n)}+h_{n} \rightarrow \gamma_{0}^{(0)} \in[0, \kappa]$. Consequently, for arbitrary $\epsilon>0$ there exists $n(\epsilon)$ such that

$$
\begin{aligned}
& \left|\gamma^{(m)}+h_{m}-\gamma^{(p)}-h_{p}\right|<\left|\gamma_{0}^{(m)}+h_{m}-\gamma_{0}^{(p)}-h_{p}\right| \\
& +\left|\gamma^{(m) i}-\gamma^{(p) i}\right|<\mid<\frac{\epsilon}{2}+\frac{\epsilon}{2}=\epsilon
\end{aligned}
$$

if $m, p>n(\epsilon)$. That is, if we fix $i \in \mathbb{Z}$, then $\left\{\gamma_{i}^{(n)}+h_{n}\right\}_{n}$ is a Cauchy sequence, and hence $\gamma_{i}^{(n)}+h_{n} \rightarrow \gamma_{i}^{(0)}, i \in \mathbb{Z}$. Furthermore, by (8) the convergence is uniform in $i$ and $\gamma_{i+1}^{(0)} \geq \gamma_{i}^{(0)}, i \in \mathbb{Z}$. Finally, the condition $m\left(\gamma^{(n)}\right)=m(\gamma)$, and Lemma 4 imply that $m\left(\gamma^{(0)}\right) \leq n_{0} m(\gamma)<\infty$. It is obvious that $\left|\gamma_{i}^{(0)}\right| \rightarrow \infty$. Hence, $\gamma^{0} \in \Gamma$. Assume that $\theta^{0}$ is 
the support of $\gamma^{(0)}$. Since $\gamma^{(n) j}$ are equipotentially almost periodic and $\left|\gamma_{i}^{(n) j}-\gamma_{i}^{(0)}\right| \rightarrow 0$ uniformly in $i$ as $n \rightarrow \infty, j \in \mathbb{Z}$, one can show that $\gamma^{(0) j}, j \in \mathbb{Z}$, are equipotentially almost periodic in a similar manner as in the proof of the theorem on almost periodicity of a limit function [21]. Consequently, $\theta^{(0)}-$ is a Wexler sequence.

The theorem is proved.

Theorem $2 \theta \in \Theta$ is a Wexler sequence if and only if it satisfies the Bohner property.

Proof. Necessity is proved by Theorem 1.

Sufficiency. Assume that $\theta \in \Theta$ is not a Wexler sequence. Then $\theta^{j}, j \in \mathbb{Z}$, are not equipotentially almost periodic, and by Lemma 3 there exists a number $\epsilon_{0}$ and a sequence of sections $I_{n}=\left[h_{n}-l_{n}, h_{n}+l_{n}\right], n \in \mathbb{N}$, where $l_{1}$ is arbitrary, $l_{n}>\max _{m<n}\left|h_{m}\right|$ and the following inequality

$$
\sup _{q, k \in \mathbb{Z}}\left|\theta_{k}^{q}-\xi\right| \geq \epsilon_{0}, \xi \in \cup_{n} I_{n}
$$

is valid. Consider a sequence of shifts $\theta+h_{n}, n \in \mathbb{N}$, and denote $h^{\prime}=\left\{h_{n}\right\}$. For arbitrary $m, p \in N, m>p$, we have that $h_{m}-h_{p} \in I_{m}$ and $\sup _{i, j \in \mathbb{Z}}\left|\theta_{i}^{j}-\left(h_{m}-h_{p}\right)\right| \geq \epsilon_{0}$ or

$$
\left.\sup _{i, j \in \mathbb{Z}} \mid \theta_{i}+h_{p}-\theta_{i+j}-h_{m}\right) \mid \geq \epsilon_{0}
$$

The last inequality means that $\theta+h_{m}$ is not in the $\epsilon_{0}-$ neighborhood of $\theta+h_{p}$. Assume that there exists a subsequence $h \subset h^{\prime}$ such that $\theta+h_{n_{k}}$ convergens to $\theta^{(0)} \in \Theta$ uniformly in $B^{s}$ - topology. Then there exist numbers $n_{m}$ and $n_{p}, n_{m}>n_{p}$, such that $\left(\theta+h_{n_{p}}\right) \frac{\epsilon_{0}}{2} \theta^{(0)}$ and $\left(\theta+h_{n_{m}}\right) \frac{\epsilon_{0}}{2} \theta^{(0)}$. By Lemma 2, $\left(\theta+h_{n_{p}}\right) \epsilon_{0}\left(\theta+h_{n_{m}}\right)$. The contradiction proves the theorem.

Subsequences $h$ and $g$ are common subsequences of sequences $h^{\prime}$ and $g^{\prime}$ if $h_{n}=h_{n(k)}^{\prime}$ and $g_{n}=g_{n(k)}^{\prime}$ for some given function $n(k)$ [21]. The following theorem is an analogue of Theorem 1.17 from [21], and we shall follow the proof of the theorem presented there.

Theorem 3 A sequence $\theta \in \Theta$ is a Wexler sequence if and only if for arbitrary $h^{\prime}$ and $g^{\prime}$ there exist common subsequences $h \subset h^{\prime}$ and $g \subset g^{\prime}$ such that

$$
Q_{h+g} \theta=Q_{h} Q_{g} \theta
$$


Proof.

Necessity. Assume that $\theta$ is a Wexler sequence. By the previous theorem there exists a subsequence $g^{\prime \prime} \subset g^{\prime}$ such that $Q_{g^{\prime \prime}} \theta$ exists and the limit is a Wexler sequence. Denote $\eta=Q_{g^{\prime \prime}} \theta$. Moreover, if $h^{\prime \prime} \subset h^{\prime}$ is common with $g^{\prime \prime}$, then one can find a sequence $h^{\prime \prime \prime} \subset h^{\prime \prime}$ such that $\mu=Q_{h^{\prime \prime \prime}} \eta$ is a Wexler sequence. If $g^{\prime \prime \prime} \subset g^{\prime \prime}$ is common with $h^{\prime \prime \prime}$ then there exist common subsequences $h \subset h^{\prime \prime \prime}, g \subset g^{\prime \prime \prime}$ such that $Q_{g+h} \theta=\zeta$. Since $h \subset h^{\prime \prime}, g \subset g^{\prime \prime}, \quad Q_{g} \theta=\eta, Q_{h} \eta=\mu$. Thus, if $\epsilon>0$ is fixed, then for sufficiently large $n$ we have $\zeta \epsilon\left(\theta+h_{n}+g_{n}\right), \quad \eta \epsilon\left(\theta+g_{n}\right)$, and $\mu \epsilon\left(\eta+h_{n}\right)$. Using Lemma 2, we can conclude that $\zeta 3 \epsilon \mu$. Hence, $\zeta=\mu$, as $\epsilon$ is arbitrarily small.

Sufficiency. Suppose that $h^{\prime}$ is the given sequence. Taking $g^{\prime}=0$ we see that the condition $Q_{g+h}=Q_{g} Q_{h} \theta$ implies that $Q_{h} \theta$ exists, and hence by Theorem 2 the sequence $\theta$ is a Wexler sequence. The theorem is proved.

\section{Bohr-Wexler almost periodic functions}

Definition 7 Let $u_{1}, u_{2} \in \mathcal{P} \mathcal{C}_{r}$, and $\theta^{(1)}, \theta^{(2)}$ be the sequences of the points of discontinuity of these functions, respectively. We shall say that $u_{1}$ is $\epsilon$ - equivalent to $u_{2}$, and denote $u_{1} \epsilon u_{2}$, if $\theta^{(1)} \epsilon \theta^{(2)}$ and $\left|u_{1}(t)-u_{2}(t)\right|<\epsilon$ for all $t \in R \backslash \cup_{i}\left[\left(\theta_{i}^{(1)}-\epsilon, \theta_{i}^{(1)}+\right.\right.$ $\left.\epsilon) \cup\left(\theta_{i}^{(2)}-\epsilon, \theta_{i}^{(2)}+\epsilon\right)\right]$. We also say that $u_{1}$ belongs to the $\epsilon-$ neighborhood of $u_{2}$, and vice versa, denoting $u_{1} \in O\left(u_{2}, \epsilon\right)$ and $u_{2} \in O\left(u_{1}, \epsilon\right)$, respectively.

Definition 8 The topology defined on the basis of all $\epsilon-$ neighborhoods of functions from $\mathcal{P C}_{r}$ is called $B$ - topology. It is clear that this topology is Hausdorff.

Definition 9 A number $\tau$ is an $\epsilon$ - translation number of $\phi \in \mathcal{P C}_{r}$ if $\phi(t+\tau) \epsilon$ $O(\phi(t), \epsilon)$.

Definition 10 A function $\phi \in \mathcal{P C}_{r}$ is a Bohr-Wexler almost periodic function, if for arbitrary $\epsilon>0$ there exists a relatively dense set of $\epsilon-$ translation numbers of $\phi$. If $\theta \in \Theta$ is a sequence of the moments of discontinuity of $\phi$, then $\theta$ is a Wexler sequence.

We shall denote by $\mathcal{B W} \mathcal{A} \mathcal{P}$ the set of all Bohr-Wexler almost periodic functions. Let $h=\left\{h_{n}\right\}$ and $T_{h} \phi \in \mathcal{P C}_{r}$ be the limit of the sequence $\phi\left(t+h_{n}\right), \phi \in \mathcal{P C}_{r}$, in $B-$ topology, if it exists. 
Definition $11 \phi \in \mathcal{P C}_{r}$ satisfies the Bohner property if every sequence $h^{\prime}$ contains a subsequence $h \subseteq h^{\prime}$ such that there exists $T_{h} \phi \in \mathcal{P C}_{r}$.

Lemma 5 If $u_{1} \epsilon_{1} u_{2}, u_{2} \epsilon_{2} u_{3}$, then $u_{1}\left(\epsilon_{1}+\epsilon_{2}\right) u_{3}$.

Proof. Lemma 1 and the relations $\theta^{(1)} \epsilon_{1} \theta^{(2)}, \theta^{(2)} \epsilon_{2} \theta^{(3)}$ imply that $\theta^{(1)}\left(\epsilon_{1}+\epsilon_{2}\right) \theta^{(3)}$. Moreover, one can easily obtain that

$$
t \in R \backslash \cup_{i}\left[\left(\theta_{i}^{(1)}-\left(\epsilon_{1}+\epsilon_{2}\right), \theta_{i}^{(1)}+\left(\epsilon_{1}+\epsilon_{2}\right)\right) \cup\left(\theta_{i}^{(3)}-\left(\epsilon_{1}+\epsilon_{2}\right), \theta_{i}^{(3)}+\left(\epsilon_{1}+\epsilon_{2}\right)\right)\right]
$$

implies that $t \in R \backslash \cup_{i}\left[\left(\theta_{i}^{(j)}-\epsilon_{j}, \theta_{i}^{(j)}+\epsilon_{j}\right) \cup\left(\theta_{i}^{(2)}-\epsilon_{j}, \theta_{i}^{(2)}+\epsilon_{j}\right)\right], j=1,3$.

That is why $\left|u_{1}(t)-u_{3}(t)\right| \leq\left|u_{1}(t)-u_{2}(t)\right|+\left|u_{2}(t)-u_{3}(t)\right|<\epsilon_{1}+\epsilon_{2}$ if (12) is valid. The lemma is proved.

Theorem $4 \phi \in \mathcal{B} \mathcal{W} \mathcal{A P}$ if and only if $\phi$ satisfies the Bohner property. $T_{h} \phi \in \mathcal{B} \mathcal{W} \mathcal{A P}$ for $\phi \in \mathcal{B W} \mathcal{A P}$, if the limit exists.

Proof. Necessity. Assume that $\phi \in \mathcal{B W} \mathcal{A P}, \theta \in \Theta$ is a sequence of the points of discontinuity of $\phi$, and $h^{\prime} \subset \mathbb{R}$ is a given sequence. By Theorem 1 there exists a subsequence of $h^{\prime}$ such that $T_{h^{\prime}} \theta=\theta^{0}$ is a Wexler sequence, and without loss of generality we assume that it is $h^{\prime}$ itself. Consider a sequence $\epsilon_{n}$ such that $\epsilon_{n} \rightarrow 0, n \rightarrow \infty$, and denote $A_{n}=\cup_{i}\left[\theta_{i}^{0}-\epsilon_{n}, \theta_{i}^{0}+\epsilon_{n}\right]$. Using the diagonal process [21], one can find $h^{(1)} \subseteq h^{\prime}$, such that $\phi\left(t+h_{n}^{(1)}\right)$ is uniformly convergent to $\phi^{(1)}$ on $A_{1}$. Then in the same way we can define a sequence $h^{(2)} \subseteq h^{(1)}$ such that $\phi\left(t+h_{n}^{(2)}\right)$ is uniformly convergent to $\phi^{(2)}$ on $A_{2}$, and so on. Obviously, $\phi^{(i+1)}=\phi^{(i)}$ on $A_{i}, A_{i} \subset A_{i+1}, \cup_{i} A_{i}=R \backslash \theta^{0}$. Consequently, $\phi\left(t+h_{n}^{(n)}\right)$ is convergent to $\phi^{0} \in \mathcal{P} \mathcal{C}_{r}$ in $B$ - topology.

Fix $\epsilon>0$. There exists $n(\epsilon)$ such that for arbitrary $n>n(\epsilon)$ the inequality $\phi(t+$ $\left.h_{n}^{(n)}\right) \frac{\epsilon}{3} \phi^{0}(t)$ is valid. If $\tau$ is an $\frac{\epsilon}{3}-$ almost period of $\phi(t)$, then $\phi\left(t+h_{n}^{(n)}+\tau\right) \frac{\epsilon}{3} \phi\left(t+h_{n}^{(n)}\right)$ and $\phi\left(t+h_{n}^{(n)}+\tau\right) \frac{\epsilon}{3} \phi^{0}(t+\tau)$. Now, using Lemma 國, one can obtain $\phi^{0}(t+\tau) \in O\left(\phi^{0}, \epsilon\right)$.

Sufficiency. Assume that $\phi \notin \mathcal{B W} \mathcal{A} \mathcal{P}$. Then, similarly to the classical case for some $\epsilon_{0}>0$, we can find a sequence of sections $I_{n}=\left[h_{n}-l_{n}, h_{n}+l_{n}\right], l_{n} \geq \max _{m<n}\left|h_{m}\right|, l_{1}-$ arbitrary, such that if $\xi \in \cup_{n} I_{n}$, then $\phi(t+\xi) \notin O\left(\phi, \epsilon_{0}\right)$. Denote $h^{\prime}=\left\{h_{n}\right\}$, and assume that there exists $h \subset h^{\prime}$ such that $T_{h^{\prime}}=\phi^{0} \in \mathcal{P C}_{r}$. Then there exists $n(\epsilon)$ such that if $m>p>n(\epsilon)$, then $\phi\left(t+h_{m}\right) \frac{\epsilon_{0}}{2} \phi^{0}(t)$, and $\phi\left(t+h_{p}\right) \frac{\epsilon_{0}}{2} \phi^{0}(t)$. By Lemma [5. 
$\phi\left(t+h_{m}\right) \epsilon_{0} \phi\left(t+h_{p}\right)$. Hence, $\phi\left(t+\left(h_{m}-h_{p}\right)\right) \epsilon_{0} \phi(t)$, but $\left(h_{m}-h_{p}\right) \in I_{m}$. The theorem is proved.

Let sequences $h^{\prime}, g^{\prime}$ be given. Subsequences $h \subset h^{\prime}, g \subset g^{\prime}$ are common subsequences of $h^{\prime}, g^{\prime}$, respectively, if $h_{n}=h_{n(k)}^{\prime}, g_{n}=g_{n(k)}^{\prime}$ for some function $n(k)$. There is analogue of Theorem 1.17 from [21, which can be proved similarly to Theorem 3 ,

Theorem $5 \phi \in \mathcal{B W} \mathcal{A P}$ if and only if for arbitrary $h^{\prime}, g^{\prime}$ there exist common subsequences $h, g$ such that $T_{h+g} \phi=T_{h} T_{g} \phi$.

Lemma 6 Assume that $f \in \mathcal{P C}_{r}$, and (3) satisfies exponential dichotomy. Then the system

$$
x^{\prime}=A(t) x+f(t)
$$

has a unique solution $x_{0}(t) \in C_{0}(t)$.

Proof. Indeed, similarly to Lemma 1 one can check that

$$
x_{0}(t)=\int_{-\infty}^{\infty} G(t, s) f(s) d s
$$

is a solution of (13) and belongs to $C_{0}(\mathbb{R})$. Assume that $x_{1}(t)$ is another solution of (13), bounded on $\mathbb{R}$. One can see that the difference $x_{1}-x_{0}$ is a continuously differentiable solution of system (3). Hence, it is a trivial solution of (3). The lemma is proved. In what follows we assume that

C5) $\theta$ is a Wexler sequence.

Using the Bohner property one can prove that the following assertion is valid.

Lemma 7 Assume that $\phi(t) \in \mathcal{A P}(\mathbb{R})$, and condition $\left(C_{5}\right)$ is valid. Then $\phi(\beta(t)) \in$ $\mathcal{B W} \mathcal{A P}$.

Assume that $\phi(t) \in \mathcal{A P}$ and $\psi(t) \in \mathcal{B} \mathcal{W} \mathcal{A P}$.

For our convenience, following [2], we shall say that

(i) a sequence $h$ is regular with respect to $\phi(t)$ if the sequence $\phi\left(t+h_{n}\right)$ is uniformly convergent on $\mathbb{R}$;

(ii) a sequence $h$ is regular with respect to $\psi(t)$ if the sequence $\psi\left(t+h_{n}\right)$ is convergent in $B$ - topology.

Let us denote by $\mathcal{L}(\phi)(\mathcal{L}(\psi))$ the set of all sequences regular with respect to $\phi(\psi)$. 


\section{Almost periodic solutions}

Lemma 8 Assume that (3) satisfies exponential dichotomy and $f(t) \in \mathcal{B W} \mathcal{A P}$. Then there is a unique solution of (13), $x_{0}(t) \in \mathcal{A P}(\mathbb{R})$, such that $\mathcal{L}\left(x_{0}\right) \subseteq \mathcal{L}(A, f)$, and $\left\|x_{0}\right\| \leq\left(\frac{K_{1}}{\sigma_{1}}+\frac{K_{2}}{\sigma_{2}}\right)\|f\|$.

Proof. By Lemma 6, the function $x_{0}(t)$ defined by (14) is a solution of (13) and $x_{0}(t) \in C_{0}(\mathbb{R})$. It is easy to verify that $\left\|x_{0}\right\| \leq\left(\frac{K_{1}}{\sigma_{1}}+\frac{K_{2}}{\sigma_{2}}\right)\|f\|$. Since every system in the hull of (3) satisfies exponential dichotomy [15], it has a unique bounded solution on $\mathbb{R}$. Let sequences $h^{\prime}$ and $g^{\prime}$ be given. There exist common sequences $h \subset h^{\prime}$ and $g \subset g^{\prime}$

such that $T_{h+g} A=T_{h} T_{g} A, T_{h+g} f=T_{h} T_{g} f$, and there exist uniform limits on compact sets $y=T_{h+g} x_{0}$ and $z=T_{h} T_{g} x_{0}$. Since $y, z \in C_{0}(\mathbb{R})$ and they are solutions of the same equation, it follows that $y=z$. By Theorem 1.17 [21], $x_{0}(t)$ is an almost periodic function. Assume that for a given sequence $h$ we have $T_{h} A=A^{*}$ and $T_{h} f=f^{*}$. We shall show that the limit $T_{h} x_{0}$ exists. Indeed, suppose, on the contrary, that the limit does not exist. Then there are two subsequences $h^{(1)} \subset h$ and $h^{(2)} \subset h$ such that

$$
\left\|x_{0}\left(t+h_{1 n}\right)-x_{0}\left(t+h_{2 n}\right)\right\| \geq \epsilon_{0}>0 \text {. }
$$

Then

$$
\left\|x_{0}\left(t+h_{1 n}-h_{2 n}\right)-x_{0}(t)\right\| \geq \epsilon_{0}>0
$$

for all $n \in \mathbb{N}$. But $T_{h_{1}-h_{2}} A=A$, and $T_{h_{1}-h_{2}} f=f$. Hence, $T_{h_{1}-h_{2}} x_{0}=x_{0}$. The theorem is proved.

Using the Bohner property again and Lemma [7, one can prove that the following lemma is valid.

Lemma 9 If $f \in \mathcal{A P}\left(\mathbb{R} \times G_{H}^{m}\right)$, and $\psi \in \mathcal{B W} \mathcal{A P}, \psi: R \rightarrow G_{H}$, then $F_{\theta}(\psi(t)) \in$ $\mathcal{B W} \mathcal{A P}$ and $\mathcal{L}\left(F_{\theta}(\psi(t))\right) \subseteq \mathcal{L}(f, \psi)$

Theorem 6 Assume that conditions $(C 1)-(C 5)$ are valid, and

$$
\operatorname{lm}\left(\frac{K_{1}}{\sigma_{1}}+\frac{K_{2}}{\sigma_{2}}\right)<1
$$

Then there exists a unique solution of (1), $\phi(t) \in \mathcal{A P}(\mathbb{R})$, such that $\mathcal{L}(\phi) \subseteq$ $\mathcal{L}(A, f, \beta)$. 
Proof. Let $\Psi=\{\psi \in \mathcal{A P}(\mathbb{R}) \mid \mathcal{L}(\psi) \subseteq \mathcal{L}(\mathcal{A}, \mathcal{F}, \beta)\}$ be a complete metric space with the $\sup -$ norm $\|\cdot\|_{0}$. Define an operator $\Pi$ on $\Psi$ such that

$$
\Pi(\psi(t))=\int_{-\infty}^{\infty} G(t, s) F_{\theta}(\psi(s)) d s .
$$

Lemma 9 implies that $F_{\theta}(\psi(s)) \in \Psi$ and $\Pi: \Psi \rightarrow \Psi$. If $\psi_{1}, \psi_{2} \in \Psi$, then

$$
\begin{aligned}
& \left\|\Pi\left(\psi_{1}(t)\right)-\Pi\left(\psi_{2}(t)\right)\right\| \leq \| \int_{-\infty}^{t} X(t) P X^{-1}(s)\left(F_{\theta}\left(\psi_{1}(s)\right)-F_{\theta}\left(\psi_{2}(s)\right) d s \|+\right. \\
& \| \int_{t}^{\infty} X(t)(I-P) X^{-1}(s)\left(F_{\theta}\left(\psi_{1}(s)\right)-F_{\theta}\left(\psi_{2}(s)\right) d s \| \leq\right. \\
& \left.\operatorname{lm}\left(\frac{K_{1}}{\sigma_{1}}+\frac{K_{2}}{\sigma_{2}}\right) \| \psi_{1}(t)\right)-\psi_{2}(t) \|_{0} .
\end{aligned}
$$

Thus, $\Pi$ is a contractive operator and there exists a unique almost periodic solution of the equation

$$
\psi(t)=\int_{-\infty}^{\infty} G(t, s) F_{\theta}(\psi(s)) d s,
$$

which is a solution of (1). The theorem is proved.

Remark 4.1 Lemma 8 and Theorem $[$ are analogous to the assertions which were obtained in [21] for ordinary differential equations.

\section{$5 \quad$ Stability}

This section is concerned with the problem of stability of the almost periodic solution of system (11). We consider a specific initial condition when values of solutions are evaluated only at points from sequence $\theta$. This approach to the stability is natural for EPCA [35, 36]. More detailed discussion of the problem can be found in [7]. Denote by $X(t, s)=X(t) X^{-1}(s)$ the Cauchy matrix of (3). We will need the following assumptions:

$\left(C_{6}\right) \exists\{\sigma, K\} \subset R, K \geq 1, \sigma>0$, such that $\|X(t, s)\| \leq K \exp (-\sigma(t-s)), t \geq s ;$

$\left(C_{7}\right) l<\frac{\sigma}{m K}$

Assume that $p_{j} \geq 0, j=\overline{1, m}$ and denote $\tau=\max \left\{\sup _{t}\left(t-\theta_{\beta(t)-p_{j}}\right), j=\overline{1, m}\right\}>$ $0, \quad \zeta(l)=1-\exp (a \tau) K \operatorname{lm}(\sigma-a)^{-1}$, where $a \in R, 0<a<\sigma$, is fixed. 
$\left(C_{8}\right) \zeta(l)>0$.

Conditions $(C 1)-(C 7)$ and Theorem [ 6 imply that there exists a unique solution of (11), $\xi(t) \in \mathcal{A P}(\mathbb{R})$.

Fix $\epsilon>0$ and denote $L(l, \delta)=\frac{K}{\zeta(l)} \delta$, where $\delta \in R, \delta>0$. Take $\delta$ so small that $L(l, \delta)<\epsilon$. Assume that $t_{0} \in \theta$. Moreover, without any loss of generality, assume that $t_{0}=\theta_{0}=0$. Fix a sequence $\eta^{j} \in \mathbb{R}^{n}, j=\overline{1, m}, \quad \max \left\|\eta^{j}\right\|<\delta$. Denote $p^{0}=\max _{\overline{1, m}} p_{j}$, and let $\Psi_{\eta}$ be the set of all continuous functions which are defined on $\left[\theta_{-p^{0}}, \infty\right)$. And if $\psi \in \Psi_{\eta}$ then: 1) $\psi\left(\theta_{-p^{j}}\right)=\eta^{j}, j=\overline{1, m} ; \quad$ 2) $\psi(t)$ is uniformly continuous on $[0,+\infty)$; and 3) $\quad\|\psi(t)\| \leq L(l, \delta) \exp (-a t)$ if $t \geq 0$.

Consider the following EPCAG and the initial condition

$$
\begin{aligned}
& \frac{d v}{d t}=A(t) v+w\left(t, v\left(\theta_{\beta(t)-p_{1}}\right), v\left(\theta_{\beta(t)-p_{2}}\right), \ldots, v\left(\theta_{\beta(t)-p_{m}}\right)\right), \\
& v(s)=\eta^{j}, j=\overline{1, m}
\end{aligned}
$$

where

$$
\begin{aligned}
& w\left(t, v\left(\theta_{\beta(t)-p_{1}}\right), v\left(\theta_{\beta(t)-p_{2}}\right), \ldots, v\left(\theta_{\beta(t)-p_{m}}\right)\right)= \\
& f\left(t, \xi\left(\theta_{\beta(t)-p_{1}}\right)+v\left(\theta_{\beta(t)-p_{1}}\right), \xi\left(\theta_{\beta(t)-p_{2}}\right)+\right. \\
& \left.v\left(\theta_{\beta(t)-p_{2}}\right), \ldots, \xi\left(\theta_{\beta(t)-p_{m}}\right)+v\left(\theta_{\beta(t)-p_{m}}\right)\right)- \\
& f\left(t, \xi\left(\theta_{\beta(t)-p_{1}}\right), \xi\left(\theta_{\beta(t)-p_{2}}\right), \ldots, \xi\left(\theta_{\beta(t)-p_{m}}\right)\right),
\end{aligned}
$$

and $w$ satisfies $w(t, 0)=0$,

$$
\left\|w\left(t, v_{1}\right)-w\left(t, v_{2}\right)\right\| \leq l \sum_{j=1}^{m}\left\|v_{1}^{j}-v_{2}^{j}\right\|
$$

$v_{i}=\left(v_{i}^{1}, \ldots, v_{i}^{m}\right) \in R^{n m}, i=1,2$. The following definition is an adapted version of a definition from [13]

Definition 12 A function $v(t)$ is a solution of the initial value problem (15) on the interval $\left[\theta_{-p^{0}}, \infty\right)$ if the following conditions are fulfilled:

(i) $v\left(\theta_{-p_{j}}\right)=\eta^{j}, j=\overline{1, m}$;

(ii) $v(t)$ is continuous on $\left[\theta_{-p^{0}}, \infty\right)$; 
(iii) the derivative $v^{\prime}(t)$ exists at each point $t \in[0, \infty)$ with the possible exception of the points $\theta_{j}, j \geq 0$, where one-sided derivatives exist;

(iv) equation (15) is satisfied by $v(t)$ on each interval $\left[\theta_{j}, \theta_{j+1}\right), j \geq 0$.

It is obvious that $v^{\prime}(t)$ is the restriction on $[0, \infty)$ of a function from $\mathcal{P C}_{r}$ and the last definition can be used for EPCAG (11), too.

Denote $\bar{\theta}=\sup _{i}\left(\theta_{i+1}-\theta_{i}\right)$. There exists a positive number $M$ such that $\|X(t, s)\| \leq$ $M$ if $|t-s| \leq \bar{\theta}$.

Assume additionally that

$\left(C_{9}\right) \quad M \bar{\theta} m l<1$.

Theorem 7 Assume that $\left(C_{1}\right)-\left(C_{3}\right),\left(C_{5}\right)-\left(C_{9}\right)$ are valid. Then there exists a unique solution of the initial value problem (15), $v(t) \in \Psi_{\eta}$.

Proof. Similarly to Lemma 1 we can check that the initial value problem is equivalent to the following integral equation

$$
\begin{aligned}
& v(t)=X(t, 0) \eta^{0}+\int_{0}^{t} X(t, s) F_{w}(v(s)) d s, \\
& v\left(\theta_{-p_{j}}\right)=\eta^{j}, j=\overline{1, m}
\end{aligned}
$$

where $F_{w}(v(s))=w\left(s, v\left(\theta_{\beta(s)-p_{1}}\right), v\left(\theta_{\beta(s)-p_{2}}\right), \ldots, v\left(\theta_{\beta(s)-p_{m}}\right)\right)$. Define on $\Psi_{\eta}$ an operator $\Pi$ such that if $\psi \in \Psi_{\eta}$, then

$$
\Pi \psi=\left\{\begin{array}{r}
\psi, t \in\left[\theta_{-p^{j}}, 0\right] \\
X(t, 0) \eta^{0}+\int_{0}^{t} X(t, s) F_{\omega}(\psi(s)) d s, t \geq 0 .
\end{array}\right.
$$

We shall show that $\Pi: \Psi_{\eta} \rightarrow \Psi_{\eta}$. Indeed, for $t \geq 0$ it is true that

$$
\begin{gathered}
\|\Pi \psi\| \leq K \exp (-\sigma t) \delta+\int_{0}^{t} K \exp (-\sigma(t-s)) l L(l, \delta) \sum_{j=0}^{m} \exp \left(-a \theta_{\beta(s)-p_{j}}\right) d s \leq \\
\exp (-a t)\left[K \delta+\frac{m \exp (a \tau) K l L(l, \delta)}{\sigma-a}\right]=L(l, \delta) \exp (-a t) .
\end{gathered}
$$

Differentiating $\Pi \psi$ on $[0, \infty)$, it is easy to show that $[\Pi \psi]^{\prime}$ exists on $[0, \infty)$ except possibly on a countable set of isolated points of discontinuity of the first kind, and that 
it is bounded on $[0, \infty)$. Hence, $\Pi \psi$ is a uniformly continuous on $[0, \infty)$ function.

Let $\psi_{1}, \psi_{2} \in \Psi_{\eta}$. Then

$$
\left\|\Pi \psi_{1}-\Pi \psi_{2}\right\| \leq \int_{0}^{t} b \exp (-a(t-s)) l m\left\|\psi_{1}-\psi_{2}\right\|_{1} d s \leq \frac{K l m}{\sigma} \sup _{t \geq 0}\left\|\psi_{1}-\psi_{2}\right\| .
$$

Using a contraction mapping argument, one can conclude that there exists a unique fixed point $v(t, \eta)$ of the operator $\Pi: \Psi_{\eta} \rightarrow \Psi_{\eta}$ which is a solution of (15). To complete the proof we should show that there exists no solution of the problem out of $\Psi_{\eta}$.

Consider first the interval $\left[\theta_{0}, \theta_{1}\right]$. Assume that on the interval (15) has two different solutions $v_{1}, v_{2}$ of the problem. Obviously, their difference $w=v_{1}-v_{2}$ is again a solution of the equation. Denote $\bar{m}=\max _{\left[\theta_{0}, \theta_{1}\right]}\|w(t)\|$, and assume, on contrary, that $m>0$. We have that on the interval

$$
\|w(t)\|=\left\|\int_{0}^{t} X(t, s) F_{w}(w(s)) d s\right\| \leq M l \bar{\theta} m \bar{m} .
$$

The last inequality contradicts condition $\left(C_{9}\right)$. Now, using induction, one can easily prove the uniqueness for all $t \geq 0$. The theorem is proved.

Denote $\phi=\left\{\phi^{j}\right\}, j=\overline{1, m}$, a sequence of vectors from $\mathbb{R}^{n}$. Let $x(t, \phi)$ be a solution of (11) such that $x\left(\theta_{-p^{j}}, \phi\right)=\phi^{j}, j=\overline{1, m}$.

Definition 13 The almost periodic solution $\xi(t)$ of (11) is said to be exponentially stable if there exists a number $a \in R, a>0$, such that for every $\epsilon>0$ there exists a number $\delta=\delta(\epsilon)$, such that the inequality $\max _{j=\overline{1, m}}\left\|\phi^{j}-\xi\left(\theta_{-p^{j}}\right)\right\|<\delta$ implies $\|x(t, \phi)-\xi(t)\|<\epsilon \exp (-a t))$ for all $t \geq 0$.

Consider now a solution $x(t, \phi)$ of (10) such that $\max _{j=1, m}\left\|\phi^{j}-\xi\left(\theta_{-p^{j}}\right)\right\|<\delta$. Since the solution $v(t)$ of the equation (15), satisfying $v\left(\theta_{-p^{j}}\right)=\phi^{j}-\xi\left(\theta_{-p^{j}}\right), j=\overline{1, m}$, exists, $x(t, \phi)=\xi(t)+v(t), t \in\left[\theta_{-p^{0}}, \infty\right)$, and $x(t, \phi)$ is uniquely continuable to $\infty$. Thus, the following theorem is proved.

Theorem 8 Assume that $\left(C_{1}\right)-\left(C_{3}\right),\left(C_{5}\right)-\left(C_{8}\right)$ are valid. Then the almost periodic solution $\xi(t)$ of (1) is exponentially stable. 


\section{The Example: positive almost periodic solutions of the logistic differential equation}

In 32 G. Seifert applied the reduction to discrete equations to the following logistic equation $\dot{N(t)}=N(t)(a(t)-f(N([t]))), t>0$, with positive coefficient $a(t)$. In his paper the conditions on the equation which guarantee the existence of an asymptotically stable almost periodic solution were found, and it was proposed to solve a similar problem for an equation of the following type

$$
N^{\prime}(t)=N(t)\left(a(t)-\sum_{j=0}^{n-1} f_{j}(N([t-j]))\right), n>1
$$

In what follows we propose a particular solution of the problem.

Consider the problem of existence of positive almost periodic solutions of the following equation

$$
x^{\prime}(t)=x(t)\left[a(t)-f\left(x\left(\left[t-p_{1}\right]\right), x\left(\left[t-p_{2}\right]\right), \ldots, x\left(\left[t-p_{m}\right]\right)\right) d s,\right.
$$

where $x \in \mathbb{R}$, integers $p_{j}, j=\overline{1, m}$, are fixed, $a(t) \in \mathcal{A P}(\mathbb{R}), a(t)>0, t \in \mathbb{R}$. We also assume that $f(0)=0, f$ is positive for positive values of arguments and it is Lipshitzian with positive constant $l$ in all arguments.It is known that $\theta_{i}=i, i \in \mathbb{Z}$, is a Wexler sequence [17, 31]. One can easily check that (17) is of type (18).

Denote

$$
M(a)=\lim _{T \rightarrow \infty} \frac{1}{T} \int_{-T}^{T} a(s) d s .
$$

Assume that $M(a)>0$. Using the Strengthened Mean Value Theorem [10], one can easily verify that there exist positive constants $K, \sigma$, such that

$$
\exp \left(\int_{s}^{t} a(u) d u\right) \leq K \exp (\sigma(t-s)), t \leq s
$$

By Lemma 1 the equivalent integral equation is

$$
x(t)=\int_{t}^{\infty} \exp \left(\int_{s}^{t} a(u) d u\right) x(s) f\left(x\left(\left[s-p_{1}\right]\right), x\left(\left[s-p_{2}\right]\right), \ldots, x\left(\left[s-p_{m}\right]\right)\right) d s .
$$

Fix $H \in \mathbb{R}, 0<H$, and denote $G=\left\{\left(z_{0}, z_{1}, z_{2}, \ldots, z_{m}\right) \in \mathbb{R}^{k+1} \mid z_{j} \leq H, j=\right.$ $\overline{0, m}\}$. Denote $\mu=\sup _{G} z_{0} f\left(z_{1}, z_{2}, \ldots, z_{m}\right)$. We assume that $0<\mu$. Consider the set of functions $\Psi=\{\psi \in \mathcal{A P}(\mathbb{R}) \mid 0 \leq \psi(t) \leq H, t \in \mathbb{R}$. 
Define an operator $\Pi$ on the set $\Psi$ such that if $\psi \in \Psi$ then

$$
\Pi \psi=\int_{t}^{\infty} \exp \left(\int_{s}^{t} a(u) d u\right) \psi(s) f\left(\psi\left(\left[s-p_{1}\right]\right), \psi\left(\left[s-p_{2}\right]\right), \ldots, \psi\left(\left[s-p_{k}\right]\right)\right) d s .
$$

We have that $\Pi \psi(t) \leq \frac{K \mu}{\sigma}$. If we assume that $\frac{K \mu}{\sigma} \leq H$ and $\frac{K}{\sigma}[l H+\mu]<1$, then in the same way as in Theorem [6 existence of a solution $\psi^{0}(t) \in \Psi$ can be proved.

\section{References}

[1] A. R. Aftabizadeh, J. Wiener and J.- M. Xu, Oscillatory and periodic solutions of delay differential equations with piecewise constant argument, Proc. Amer. Math. Soc. 99 (1987), 673-679.

[2] L. Amerio and G. Prouse, "Almost-periodic functions and functional equations," Van Nostrand Reinhold Company, new York, 1971.

[3] M. U. Akhmetov and N. A. Perestyuk, Periodic and almost-periodic solutions of strongly nonlinear impulse systems, J. Appl. Math. Mech., 56 (1992), 829-837.

[4] M. U. Akhmetov and N. A. Perestyuk, Almost-periodic solutions of nonlinear impulse systems, Ukrainian Math. J., 41 (1989), 259-263.

[5] M. U. Akhmetov, N. A. Perestyuk and A.M. Samoilenko, "Almost-periodic solutions of differential equations with impulse action," (Russian) Akad. Nauk Ukrain. SSR Inst., Mat. Preprint, 1983, no. 26, 49 pp.

[6] M. U. Akhmet, Existence and stability of almost-periodic solutions of quasi-linear differential equations with deviating argument, Applied mathematics Letters, 17 (2004), 1177-1181.

[7] M. U. Akhmet,Integral manifolds of differential equations with piecewise constant argument of generalized type, Nonlinear Analysis, Theory, methods and applications, (in press).

[8] A. Alonso, and J. Hong, Ergodic type solutions of differential equations with piecewise constant arguments, Int. J. Math. Math. Sci., 28 (2001), 609-619. 
[9] A. Alonso, J. Hong and R. Obaya, Almost-periodic type solutions of differential equations with piecewise constant argument via almost periodic type sequences, Appl. Math. Lett., 13 (2000), 131-137.

[10] H. Bohr, "Almost-periodic functions," Chelsea Publishing Company, New York, 1951.

[11] S. Busenberg and K. Cooke, "Vertically transmitted diseases, Models and dynamics. Biomathematics, 23,"Springer-Verlag, Berlin, 1993.

[12] T.A. Burton and Tetsuo Furumochi, Fixed points and problems in stability theory for ordinary and functional differential equations, Dynamic Systems and Appl., 10 (2001), 89-116.

[13] K. L. Cooke and J. Wiener, Retarded differential equations with piecewisw constant delays, J. Math. Anal. Appl., 99 (1984), 265-297.

[14] C. Corduneanu, Almost periodic functions, Interscience Publishers, New York, 1961.

[15] W.A. Coppel, "Dichotomies in stability theory," Lecture notes in mathematics, Springer-Verlag, Berlin, Heidelberg, New York, 1978.

[16] S. Doss and S.K. Nasr, On the functional equation $y^{\prime}=f(x, y(x), y(x+h)), h>0$, Amer. Journ. Math., 75 (1953),713 - 716.

[17] A. Halanay and D. Wexler "Qualitative theory of impulsive systems," (Romanian) Republici Socialiste Romania, Bucuresti, 1968.

[18] J. Hale, "Functional differential equations," Springer-Verlag, New York, Heidelberg, Berlin, 1971.

[19] K. Gopalsamy, "Stability and oscillations in delay differential equations," Kluwer Academic Publishers Group, Dordrecht, 1992.

[20] A. F. Filippov, "Differential equations with discontinuous righthand sides," Mathematics and its Applications (Soviet Series), 18. Kluwer Academic Publishers Group, Dordrecht, 1988. 
[21] A.M. Fink, "Almost-periodic differential quations, " Lecture notes in mathematics, Springer-Verlag, Berlin, Heidelberg, New York, 1974.

[22] L.E. El'sgol'ts, "Introduction to the theory of differential equations with deviating arguments, " Holden-Day, Inc, San Francisco, London, Amsterdam, 1966.

[23] A.N. Kolmogorov, On the Skorohod convergence (Russian. English summary), Teor. Veroyatnost. i Primenen., 1 (1956), 239-247.

[24] M. A. Krasnosel'skii, V. Sh. Burd and Yu. S. Kolesov, "Nonlinear almost periodic oscillations," John Wiley Sons, New York-Toronto, 1973.

[25] T. Küpper and R. Yuan, On quasi-periodic solutions of differential equations with piecewise constant argument, J. Math. Anal. Appl., 267 (2002), 173-193.

[26] Yu. A. Kuznetsov, "Elements of Applied Bifurcation Theory," Springer-Verlag, New-York, Berlin, Heidelberg, 1995.

[27] R.M. May and G.F. Oster, Bifurcations and dynamic complexity in simple ecological models, Amer. Natural, 110 (1976) 573-599.

[28] G. Papaschinopoulos, Some results concerning a class of differential equations with piecewise constant argument, Math. Nachr. 166 (1994), 193-206.

[29] G. Papaschinopoulos, Linearisation near the integral manifold for a system of differential equations with piecewise constant argument, J. of Math. Anal. and Appl. (1997), 317-333.

[30] S.M. Shah and J. Wiener, Advanced differential equations with piecewise constant argument deviations, Internat. J. Math. Sci, 6 (1983), 671-703.

[31] A. M. Samoilenko and N. A. Perestyuk, "Impulsive Differential Equations," World Scientific, Singapore, 1995.

[32] G. Seifert, Almost periodic solutions of certain differential equations with piecewise constant delays and almost periodic time dependence, J. Differential equations, 164 (2000), 451-458. 
[33] A.V. Skorokhod, Limit theorems for random processes, Theory Probab. Appl., 39 (1994),289-319,

[34] V.E. Slyusarchuk, Bounded solutions of impulsive systems, Differentsial'nye Uravneniya, 19 (1983) 588-596.

[35] D. Wexler, Solutions périodiques et presque-périodiques des systémes d'équations différetielles linéaires en distributions, J. Differential Equations., 2 (1966), 12-32.

[36] J. Wiener, Generalized solutions of functional differential equations, World Scientific, Singapore (1993).

[37] J. Wiener and V. Lakshmikantham, A damped oscillator with piecewise constant time delay, Nonlinear Stud., 7 (2000), 78-84.

[38] Yuan Rong, On the spectrum of almost periodic solution of second order scalar functional differential equations with piecewise constant argument, J. Math. Anal. Appl., 303 (2005) 103-118.

[39] Muroya, Yoshiaki, Persistence, contractivity and global stability in logistic equations with piecewise constant delays, J. Math. Anal. Appl., 270 (2002), 602-635.

[40] C. Zhang, "Almost periodic type functions and ergodicity, Science press," Beijing/New York, Kluwer Academic Publishers, Dordecht/ Boston/ London, 2003. 